\title{
FUNDAMENTALISMOS ISLÁMICOS Y JUDEOFOBIA: CONEXIONES SUDAMERICANAS
}

\author{
Isaac Caro \\ Instituto de Ciencias Políticas, \\ Universidad Alberto Hurtado \\ Universidad Arturo Prat \\ isaac.caro@gmail.com
}

\begin{abstract}
RESUMEN
Esta presentación corresponde a un proyecto FONDECYT ${ }^{1}$, en donde se examina la presencia - real, potencial y simbólica -, de movimientos fundamentalistas islámicos en Sudamérica, en el periodo 1992-2006, a partir de cuatro hitos o momentos emblemáticos. Estos hitos corresponden a atentados terroristas reivindicados por distintas organizaciones islamistas, que tuvieron como objetivos: a) la Embajada de Israel en Buenos Aires (17 de marzo de 1992); b) el edificio compartido por la Asociación Mutual Israelita Argentina - AMIA - y la Delegación de Asociaciones Israelitas Argentinas - DAIA -, también en Buenos Aires (18 de julio de 1994); c) los edificios del World Trade Center en Nueva York y del Pentágono en Washington (11 de septiembre de 2001); d) la red ferroviaria y la Estación Atocha de Madrid (11 de marzo de 2004). Aquí debemos diferenciar entre hitos globales en el caso de los dos últimos - e hitos regionales - en lo que se refiere a los dos primeros.

Al mismo tiempo, se establece una periodización, considerando dos etapas: la primera va desde el atentado de 1992 hasta septiembre de 2001, en que las denuncias sobre la presencia islamista en Sudamérica apuntan casi exclusivamente a movimientos islamistas chiítas, representados por Hezbolá, con apoyo de Irán. La segunda se extiende desde esa fecha hasta nuestros días, aunque para propósitos de esta investigación se considerará como cierre noviembre de 2006, cuando se dan a conocer avances judiciales en la causa por el atentado contra la AMIA-DAIA. En este segundo subperíodo, a la presencia de Hezbolá, se unen las denuncias sobre las operaciones de Al Qaeda y de grupos vinculados con Jamaat Tabligh (propagación) en distintos países sudamericanos, al tiempo que la Triple Frontera de Argentina, Brasil y Paraguay adquiere un carácter de "espacio transnacional".
\end{abstract}

PALABRAS CLAVE:

Fundamentalismo - Judeofobia - Sudamérica - Religión.

\section{ABSTRACT}

This paper corresponds to a FONDECYT project, that examines the presence of Islamite movements in South America, in the period 1999-2006, considering four main historical moments. These emblematic moments correspond to terrorist attacks that were related with different islamist organizations. The targets of these attacks were the following: a) the Israel Embassy in Buenos Aires (March 17 ${ }^{\text {th }}, 1992$ ); b) the building belonging to AMIA and DAIA, also in Buenos Aires July 18t, 1994); c) the World Trade Center in New York and the Pentagon in Washington DC (September 11 1 th 2001); d) the railroad network and the Atocha rail station in Madrid (March 11, 2004).

At the same time, we formulate a periodization that considers two stages: the first is between the 1992' attack and September 2001; in this period the reports about the islamist presence in South America argue the participation of Shiite movements, particularly Hizbullah, supported by Iran. The second stage is between 2001 and the present times, and is characterized by some judicial developments about the AMIA-DAIA case. In this second stage, there

1 Proyecto № 1080024, concurso de 2008: Conexiones sudamericanas del fundamentalismo islámico: 1992-2006. Coinvestigadora de este proyecto es Isabel Rodríguez, académica de la Universidad Alberto Hurtado. 
are reports about the presence of other islamist movements, like Al-Qaeda and groups related with Jamaat Tablig. Furthermore, the triborder of Argentina, Brazil and Paraguay acquires an attribute of "transnational place".

KEY WORDS

Fundamentalism - Judeophobia - South America - Religion

\section{Introducción ${ }^{2}$}

En este estudio examinamos la presencia - real, potencial y simbólica - de movimientos fundamentalistas islámicos en Sudamérica, en el periodo 1992-2006, a partir de cuatro hitos o momentos emblemáticos. Estos hitos corresponden a atentados terroristas reivindicados por distintas organizaciones islamistas, que tuvieron como objetivos: a) la Embajada de Israel en Buenos Aires (17 de marzo de 1992); b) el edificio compartido por la Asociación Mutual Israelita Argentina - AMIA - y la Delegación de Asociaciones Israelitas Argentinas - DAIA -, también en Buenos Aires (18 de julio de 1994); c) los edificios del World Trade Center en Nueva York y del Pentágono en Washington (11 de septiembre de 2001); d) la red ferroviaria y la Estación Atocha de Madrid (11 de marzo de 2004).

Al mismo tiempo, establecemos una periodización, considerando dos etapas: la primera va desde el atentado de 1992 hasta septiembre de 2001, en que las denuncias sobre la presencia islamista en Sudamérica apuntan casi exclusivamente a movimientos islamistas chí́tas, representados por Hezbolá, con apoyo de Irán. La segunda se extiende desde esa fecha hasta nuestros días, aunque para propósitos de esta investigación se considerará como cierre el mes de noviembre de 2006, cuando se dan a conocer algunos avances judiciales en la causa por el atentado contra la AMIA-DAIA. En este segundo subperiodo, a la presencia de Hezbolá, se unen las denuncias sobre las operaciones de Al Qaeda y de grupos vinculados con Jamaat Tabligh (propagación) en distintos países latinoamericanos. Indicaremos el 11 de septiembre de 2001 como un hito internacional que marcará una forma distinta de considerar, denunciar y reflexionar sobre la existencia de estos movimientos en la región. A partir de esta fecha, "cabe hablar no sólo de la reemergencia de un nuevo actor, sino que también de la afirmación y la consolidación de un fenómeno de significante importancia, que se convierte en un factor emblemático y paradigmático de los nuevos conflictos del siglo XXI" (Caro, 2002: 12). Al mismo tiempo, la Triple Frontera de Argentina, Paraguay y Brasil, se convierte en un "espacio transnacional".

Argumentamos que en relación con los cuatro momentos emblemáticos arriba señalados, estamos en presencia de diferentes formas de fundamentalismos islámicos. En lo que se refiere a Hezbolá, correspondiente a un "islamismo chiíta" apoyado por Irán, su presencia y participación latinoamericana es una extensión del conflicto con Israel, con el sionismo y con el mundo judío, conflicto que opera más allá de las fronteras regionales del Medio Oriente. En el caso de la presencia de Al Qaeda se trata de de un "islamismo sunnita jihadista global", que busca una guerra santa contra Occidente, Estados Unidos y sus aliados. América Latina es visualizada también como una prolongación de Occidente y de Estados Unidos, sin que en este caso el conflicto árabe/israelí tenga una connotación decisiva como en el caso anterior. En cuanto a la presencia de Jamaat Tabligh en Sudamérica, que corresponde a un "islamismo sunita misionero", su objetivo fundamental es de carácter proselitista, esto es, la preservación de la identidad musulmana y del orden moral islámico frente a los no

\footnotetext{
${ }^{2}$ Nota conceptual: En el marco de esta presentación utilizaremos en forma indistinta los términos "fundamentalismo islámico", "islamismo" e "islam político". Para una discusión sobre estos conceptos, ver Caro, 2002, p. 20.
} 
creyentes, por una parte, y la búsqueda de "nuevos musulmanes" a través de la conversión al islam por parte de población sudamericana.

\section{Hitos principales en la presencia de movimientos islamistas}

El primer hito que nos indica la presencia de movimientos islamistas radicales en la región latinoamericana se produce el 17 de marzo de 1992, fecha del ataque contra la Embajada de Israel en Buenos Aires. Las evidencias apuntaban y apuntan a establecer una correlación entre este atentado y el conflicto árabe - israelí, en especial en lo referente a dos conjuntos de acontecimientos: los del sur del Líbano y la cuestión palestina. El ataque se produce tras la intervención de Israel en el Líbano y, más particularmente, de la muerte por fuerzas israelíes del líder de Hezbolá, Abbas al-Musawi (19521992). De hecho, en la reivindicación del ataque, Hezbolá señaló que aquel había sido ejecutado en memoria de Musawi (Schwartz, 1995: 26; Caro, 2002: 147). Por su parte, el atentado contra la Embajada israelí tiene lugar cinco meses después del inicio de la Conferencia de Paz de Madrid, la que había sido convocada por Estados Unidos y la Unión Soviética, en octubre de 1991, para encontrar una solución negociada al enfrentamiento de Israel con Jordania, Líbano, Siria y los palestinos. Oponiéndose al proceso político, Irán promovió una conferencia paralela, al cierre de la cual Hezbolá y otros grupos formaron un "frente de rechazo", el que tenía por finalidad apoyar la creación de un Estado palestino, rechazando la existencia del Estado de Israel.

El segundo hito acontece el 18 de julio de 1994, cuando ocurre el atentado contra el edificio de la AMIA-DAIA. Tanto las autoridades argentinas como el Departamento de Estado de Estados Unidos calificaron este ataque como "el acto terrorista más importante de la historia argentina y el ataque más grave contra una comunidad judía desde la Segunda Guerra Mundial" (Caro, 2002: 148). A partir de este ataque, se comienza a denunciar la existencia de personas y grupos vinculados con Hezbolá a través de la denominada "Triple Frontera". Se trata de un espacio fronterizo entre Argentina, Paraguay y Brasil, que incluye a Ciudad del Este (Paraguay), Foz do Iguaçu (Brasil) y Puerto Iguazú (Argentina) (Federal Research Division, 2003:9; Montenegro, Giménez, 2006: 24).

Desde 1992 hasta 2001, las denuncias sobre la presencia de grupos islamistas radicales en Sudamérica apuntan casi exclusivamente a la responsabilidad de Hezbolá, de Irán y también de Siria. Informes de inteligencia, tanto de Estados Unidos como de Israel, así como las investigaciones en el marco de la justicia argentina, se inclinaban por esta pista. Informes de la Oficina de Coordinación para Contraterrorismo del Departamento de Estado de Estados Unidos (Patterns of Global Terrorism 2002) publicados de 1994 a 1998, atribuyen a la organización chí́ta libanesa la autoría de los dos atentados realizados en Buenos Aires. Según estos informes, el primero de los ataques fue adjudicado por la organización Jihad Islámico, brazo armado de Hezbolá, en tanto que el segundo, por Ansar Allah ("Seguidores de Dios"), perteneciente al mismo movimiento (US Department of State, 1995, 1996, 1997, 1998).

En el marco de la investigación judicial argentina sobre el atentado contra la Embajada de Israel en Buenos Aires, el embajador argentino en el Líbano en momentos del hecho, Juan Angel Faraldo, ratificó una serie de cables emitidos por la Embajada a su cargo, donde se daba cuenta de que el líder de Hezbolá, Hussein Fadlallah, le habría manifestado personalmente su oposición al sionismo y que la mantención de esta milicia armada se correspondía con la oposición a Israel. El informe judicial de 1999 (Canevari, 1999) es categórico sobre la responsabilidad de Irán y de Hezbolá en la organización y realización del ataque contra la Embajada israelí en Buenos Aires. En cuanto a la causa AMIA-DAIA, el juez Galeano, - hoy apartado y procesado por obstrucción a la justicia -, siguiendo las declaraciones de testigos iraníes no identificados en Alemania, había establecido en 1998 que este CUADERNOS JUDAICOS-ISSN: 0718-8749 
ataque fue planificado y decidido en Teherán, como represalia por la suspensión argentina de envío de tecnología nuclear a Irán (La Nación Line, 1998/05/16). Esto llevó al gobierno argentino a solicitar el retiro de siete diplomáticos iraníes acreditados en Buenos Aires y a un casi rompimiento de relaciones diplomáticas con Irán.

Hay que señalar que la cancelación de un embarque nuclear a Irán como móvil del ataque contra la AMIA - DAIA era una tesis que se manejaba con fuerza en el año 1998. Fuentes del gobierno argentino, de la SIDE y de la justicia confirmaron que en el marco de la investigación llevada por el juez Galeano se había revelado que este atentado había sido decidido en 1992, en Irán, como represalia contra Argentina por la cancelación de un embarque de exportaciones de tecnología nuclear. La exportación de tecnología para completar el ciclo de uranio había sido resuelta en dos contratos bilaterales de octubre de 1988, los que fueron anulados por presión Estados Unidos e Israel (La Nación Line, 1998/05/16).

¿Y qué pasa con la conexión siria? Las denuncias van en dos direcciones principales. Primero, según informes de la Cámara de Representantes de Estados Unidos, anteriores al 2001 (Bodansky, Forrest, 1994), como resultado de la cooperación de Siria con los principales carteles de droga latinoamericanos, grandes cantidades de dólares falsificados habrían ingresado a América Latina. Este flujo de drogas y dinero falsificado sirve, - dice el informe -, para sostener y financiar el incremento bélico de las "redes terroristas islámicas" con presencia en México, Argentina y Estados Unidos. Los informes agregan que el núcleo de este sistema de apoyo clandestino es un complejo de compañías comerciales, las que proveen apoyo clandestino logístico, financiero y comunicacional a estas redes.

Segundo, de acuerdo a fuentes de inteligencia estadounidenses, tanto Siria como Libia habían provisto ayuda financiera para la campaña presidencial argentina de Carlos Saúl Menem en 1989. Después de que Irak invadió Kuwait (agosto de 1990), Menem habría prometido al presidente sirio, Hafez al-Assad, que Siria heredaría la participación iraquí en el proyecto del misil balístico Cóndor II y sería también un recipiente privilegiado de tecnología nuclear argentina. Sin embargo, estas promesas no se cumplieron: debido a la presión diplomática de Estados Unidos, Argentina debió cancelar el proyecto del misil Cóndor y firmar el Tratado de Proliferación No Nuclear. Assad se habría sentido traicionado, decidiendo eliminar a Argentina de los objetivos prohibidos para Hezbolá (Escudé, Gurevich, 2003: 130) ${ }^{3}$.

El tercer hito en la presencia de movimientos islamistas radicales en Sudamérica ocurre con los atentados del 11 de septiembre de 2001, cuando en forma reiterada los servicios secretos de Estados Unidos empezaron a denunciar la presencia de Al-Qaeda, tanto en la Triple Frontera como en otros países sudamericanos. Un completo informe preparado en Estados Unidos el año 2003 (Federal Research Division, 2003) estableció que en esa zona existían evidencias sobre actividades y participación de "grupos terroristas islámicos", que incluían Al Yama Al Islamia (Asociación Islámica) de Egipto, a Hamas, a Hezbolá y a Al-Qaeda, entre otros. Estas actividades se referían, según el informe, a lavado de dinero, tráfico de drogas y armas, recolección de fondos para estas organizaciones, contando con los nexos de parte de la población islámica que residía en la zona y que luego de los atentados del año 2001 se habría desplazado a otros centros de población árabe en América Latina.

En cuanto a la presencia de Al Qaeda en la Triple Frontera, el documento estadounidense señalaba reportajes noticiosos y de la inteligencia argentina que darían cuenta de su presencia antes de los

${ }^{3}$ Sobre el proyecto del misil Cóndor Il y la transferencia nuclear de Argentina a Irán, ver Caro, 1995: 45-55. CUADERNOS JUDAICOS-ISSN: 0718-8749 
atentados de 2001, así como de la cooperación con Hezbolá a partir de una alianza estratégica entre ambas organizaciones. Al mismo tiempo, se informaba de la supuesta existencia de una cumbre realizada por estas agrupaciones a fines de 2002 en la zona, destinada a planear ataques terroristas contra objetivos estadounidenses e israelíes en Sudamérica. Más concretamente, durante 1999 agentes de la SIDE argentina que visitaron el triángulo fronterizo informaron a los servicios de inteligencia de Estados Unidos (CIA), Israel (Mossad), Francia, Alemania, Brasil y Paraguay, de la presencia de miembros vinculados con Al Qaeda y de que tenían evidencias de una colaboración conjunta entre grupos islamistas chítas y sunitas. Entre los agentes de Al Qaeda mencionados en esa ocasión por la SIDE había una figura clave: el egipcio Al Said Hassan Hussein Mokhless, entrenado en Afganistán, integrante de Al Yama Al Islamia de Egipto, acusado de los atentados contra turistas en Luxor (diciembre de 1997) y que tendría como misión tomar contacto con simpatizantes del Hamas y Hezbolá (Santoro, 2001).

El informe de la SIDE tiene una triple importancia: a) da cuenta de la presencia de Al Qaeda en la Triple Frontera antes de los atentados de septiembre de 2001, lo que no había sido establecido por los informes de inteligencia estadounidenses; b) denuncia que "por primera vez en la historia" existen evidencias en la región de una colaboración conjunta entre grupos islamistas chiítas y sunitas, la que habría tenido lugar en Ciudad del Este y Foz de Iguaçú; c) informa que la presencia de la organización conducida por Osama Bin Laden no se limita a la Triple Frontera, sino que incluye también otras zonas sudamericanas. De hecho, Mokhless fue detenido en 1999 en la ciudad uruguaya de Chuy, fronteriza de Brasil, a partir de lo cual se empezó a investigar una conexión uruguaya de Al Qaeda en esta zona fronteriza (Preve, 1999; Federal Research Division, 2003: 36). Por su parte, la conexión chilena con la Triple Frontera fue mencionada en noviembre de 2001, cuando el gobierno chileno confirmó la existencia de redes financieras vinculadas con empresas de la zona franca de lquique pertenecientes al comerciante libanés Assad Ahmad Mohamed Bakarat, el que estaría vinculado, no con Al Qaeda, sino con Hezbolá (Caro, 2002: 156-157; Federal Research Division, 2003: 37; US Department of State, 2003).

Un cuarto hito lo constituyen los atentados realizados en España, en marzo de 2004. Al año siguiente, la prensa argentina denunció que los servicios de inteligencia locales, alertados por sus pares de España e Italia, habían detectado a cinco grupos, integrados por 26 personas, del movimiento Jamaat Tabligh, los que "ingresaron en la Argentina en los últimos siete meses con el posible objetivo de contactar y reclutar ciudadanos argentinos de la comunidad musulmana para ser entrenados en el exterior en actividades terroristas". Se informó que estas personas provenían de Pakistán, Malasia, Sudáfrica, Qatar y Egipto (Gallo, 2005).

Sin embargo, expertos en inteligencia aclararon que estos grupos no tenían ninguna relación con los atentados realizados en Buenos Aires en 1992 y 1994 y que su misión era reclutar adeptos para las filas de Al-Qaeda, buscando personas musulmanas con pasaporte argentino, de manera de no despertar sospechas. El presidente de la Organización Islámica Argentina (OIA), sheij Mahmud Aid, reconoció la presencia de miembros del movimiento Jamaat Tabligh, aunque repudió su accionar con las siguientes palabras: "No compartimos la ideología y el método de trabajo que tiene esta gente. Difunden el Islam a los no musulmanes pero no reclutando gente. Desconozco que tengan contactos con redes de terrorismo internacionales. Ellos tienen una interpretación muy particular del Islam, que la mayoría de los musulmanes en la Argentina y en el mundo no comparte" (La Nación Line, 2005/09/02a).

Paralelamente, medios de comunicación latinoamericanos (como El Diario Exterior y La República, de Argentina; Radio Cooperativa y La Nación de Chile), así como agencias internacionales (United Press International) y organizaciones judías (DAIA, AMIA), refirieron estas noticias proporcionadas por La Nación de Buenos Aires y algunos de ellos resaltaron también una conexión chilena, brasilera y CUADERNOS JUDAICOS-ISSN: 0718-8749 
uruguaya de Jamaat Tabligh. La primera habría tenido su origen en marzo de 2005 en una reunión internacional de este movimiento celebrada en territorio chileno (Gallo, 2005), en tanto que la conexión brasilera y uruguaya tendría su base en la ciudad de Chuy, ubicada en la frontera uruguayo - brasilera (La Nación Cl, 2006/10/28).

Esta noticia y el reconocimiento por parte del gobierno y de comunidades musulmanas argentinas de la existencia de esta agrupación generaron un fuerte debate interno en Argentina entre el gobierno y la oposición durante el año 2005. Sectores vinculados a la Unión Cívica Radical señalaron que ya existían antecedentes sobre la existencia de estos grupos en la Triple Frontera y que incluso éstos pudieron estar vinculados con los dos atentados. Junto con negar esta información, el gobierno señaló que se estaban realizando tareas de investigación para detectar e identificar a estos grupos. En los otros países sudamericanos, en cambio, los gobiernos se negaron a entregar información oficial al respecto (La Nación Line, 2005/09/02b).

Además de estas denuncias, continúan los informes sobre la presencia de Al Qaeda en la región y de una organización denominada Hezbolá América Latina, que estaría ligada - al menos ideológicamente - con la organización basada en el Líbano. Sobre el primer punto, en febrero de 2007, la prensa venezolana denunció la presencia de esta organización en Venezuela, donde tendría el objetivo de atacar las reservas petroleras en las zonas que suministran petróleo a Estados Unidos. Según esta información, Al Qaeda habría considerado que los objetivos terroristas no debían estar limitados a Medio Oriente y Estados Unidos, sino que debían incluir también a Canadá, Venezuela y México, puesto que estos países son abastecedores de petróleo de Estados Unidos (El Universal, 2007/02/15).

Sobre la denominada Hezbolá América Latina, se trataría de dos grupos, especialmente con presencia virtual, poseedores de sitios de Internet: uno, formado en Venezuela, y el otro, en Argentina, los que responden a la autodenominación de Hezbolá Venezuela y Hezbolá Argentina, respectivamente. El primero, aunque declara que no es parte de Hezbolá del Líbano, sostiene que asume también la "tesis yihadista" en el sentido de que el Islam está siendo víctima de una cruzada por parte de Occidente. Se declara que frente a esta situación, se deben tomar las armas para defender al Islam y a los musulmanes en una guerra contra Occidente (Hezbollah Latinoamérica, en Torres, 2006: 8-9). Hezbolah Venezuela se ha hecho visible en un momento en que la relación entre Hugo Chávez y el presidente iraní se ha convertido en asunto de interés internacional. Además, Venezuela se consideró durante mucho tiempo una de las bases del Hezbolah libanés en América Latina, especialmente la Isla Margarita, que albergaba una comunidad árabe musulmana considerable (Karmon, 2006/11/14). Hezbolá Argentina, por su parte, incluye elementos de derecha radical combinados con izquierdistas populistas; ambas tendencias manifiestan relaciones muy estrechas con sectores de la comunidad chiíta árabe local y el régimen iraní (Karmon, 2006/11/14).

\section{Consideraciones finales}

Primero, estableceremos el "11-S" como una fecha y un hito de trascendencia no sólo global, por los atentados cometidos en Estados Unidos y el inicio de una "guerra en contra del terrorismo", sino también por su significancia regional latinoamericana y, en especial, sudamericana. Es posible hablar de un antes y un después del "11-S". El discurso periodístico describe el mundo y sus espacios de manera distinta a partir de esta fecha e incluso los atentados contra la Embajada de Israel y contra la AMIA-DAIA - correspondientes al primer y al segundo hito definidos en nuestro proyecto - serán leídos con una nueva significación y connotación dentro de "una geografía global del terrorismo" (Montenegro, Giménez, 2006: 235). 
Segundo, postulamos que en relación con los cuatro momentos emblemáticos arriba señalados, estamos en presencia de diferentes formas de fundamentalismos islámicos. En lo que se refiere a los ataques en Buenos Aires, conectados con Irán y Hezbolá, se trata de un "fundamentalismo chiíta", que opera en forma más integrada y cohesionada que los islamismos sunitas y que puede adoptar formas radicales y violentas, como las eventualmente expresadas en Argentina. América Latina y, particularmente, Argentina, es asociada con Occidente, siendo al mismo tiempo una extensión del conflicto árabe/israelí. En el caso de los atentados en Estados Unidos y España, asociados con $A /$ Qaeda, se trata de un "fundamentalismo sunnita jihadista global", de carácter radical, que busca una guerra santa contra Occidente, Estados Unidos y sus aliados, en especial Israel. América Latina es visualizada también como una prolongación de Occidente y de Estados Unidos, sin que en este caso el conflicto árabe/israelí tenga una connotación decisiva. En cuanto a Jamaat Tabligh, lo que corresponde a un "fundamentalismo sunnita misionero", el objetivo central es la preservación de la identidad musulmana y del orden moral islámico frente a las fuerzas de los no creyentes, buscando la captación de "nuevos musulmanes" a través de conversión de parte de la población latinoamericana.

Tercero, postulamos que también existen marcadas diferencias en cuanto a las evidencias, denuncias y certezas sobre el accionar de estos movimientos en la región. En lo que se refiere a Al Qaeda y Jamaat Tabligh, existe un discurso periodístico y de inteligencia, que ha logrado reunir múltiples pruebas y datos, aunque no hayan conducido a causas judiciales determinantes. Este discurso ha adquirido mayor fuerza a partir del "11-S". En cambio, en lo que dice relación con la existencia de Hezbola, la situación es distinta, en la medida que existen investigaciones judiciales y elementos probatorios que demostrarían su accionar en la región sudamericana, así como su responsabilidad en los atentados contra la Embajada de Israel y contra la AMIA.

Cuarto, hay que destacar la importancia transnacional que adquiere el espacio conocido como "Triple Frontera", al asumir una nueva dimensión y connotación a partir del "11-S": el triángulo fronterizo de Argentina, Brasil y Paraguay pasa a ser considerado por la prensa internacional como un área con características propias, comienza a asumirse como un espacio transnacional, que implica flujos transnacionales de armas, drogas y todo tipo de mercancías, legales e ilegales. Antes era un lugar de intersección entre tres países, un territorio o espacio imaginado; ahora es un espacio transnacional, el eje de todas las denuncias sobre la presencia de movimientos islamistas en Sudamérica (Montenegro, Giménez, 2006: 16).

Quinto, éste es un fenómeno que tiende a ser minoritario al interior del islam, no en el sentido de que carezca de importancia regional y global, sino en el de que no compromete al conjunto del Islam, ni a las corrientes islamistas más moderadas, ni mucho menos a la población musulmana. Aquí cabe hacer una diferencia sustancial entre el Islam - en cuanto cultura, religión y civilización que ha hecho una contribución sustancial e invaluable a la humanidad - y el islamismo - entendido como un movimiento político religioso que surge al interior del Islam, que busca una aplicación de las leyes religiosas islámicas y que tiene una serie de corrientes y variedades heterogéneas, algunas contrapuestas entre sí. Esta diferenciación entre Islam e islamismo, así como la necesidad de enfatizar en la multiplicidad de "islames" posibles - existe un Islam iraní, un Islam turco, un Islam egipcio, etc. -, es un primer paso para superar un paradigma de choque civilizacional. Por el contrario, aquí afirmaremos la multidimensionalidad y pluralidad que caracteriza al Islam en general y a los fundamentalismos islámicos en particular. Esta visión es acorde con los postulados que señalan la necesidad de crear alianzas, encuentros y puentes entre civilizaciones. Partiremos del supuesto de que los grandes enfrentamientos no son aquellos que se dan entre civilizaciones, culturas o religiones distintas, sino que los que se dan al interior de una misma civilización, cultura o religión. En este CUADERNOS JUDAICOS-ISSN: 0718-8749 
sentido, visualizaremos los fundamentalismos islámicos con presencia en Sudamérica como movimientos y corrientes dispares, contradictorias, y enfrentadas con las principales comunidades árabes y musulmanas de la región latinoamericana.

№26, diciembre 2009 


\section{REFERENCIAS BIBLIOGRÁFICAS}

BODANSKY, Yossef; VAUGHN, Forrest, S. 1994. "Iran, Syria and the trail of the counterfeit money". Washington: Task Force on Terrorism \& Unconventional Warfare. US House of Representatives

. www.ict.org.il

(Consulta: 19-11-1998).

CANEVARI, Esteban Jorge. "Autos, vistos y considerando en relación con el atentado contra la sede de la Embajada de Israel en Buenos Aires". Buenos Aires: Corte Suprema de Justicia de la Nación, 10 de mayo $1999 .$.

CARO, Isaac. "América Latina-Medio Oriente: relaciones militares".EN: Fuerzas Armadas y Sociedad, Volumen X, №3, Julio - Septiembre 1995., pp. 45-55.

CARO, Isaac, Fundamentalismos islámicos. Guerra contra Occidente y América Latina. Colección Todo es Historia. Editorial Universitaria. 2002. Santiago:

ESCUDÉ, Carlos; GUREVICH, Beatriz "Limits to governability, corruption and transnational terrorism: the case of the 1992 and 1994 attacks in Buenos Aires". Estudios Interdisciplinarios de América Latina y el Caribe. Volumen 14, № 2, 2003. Tel Aviv. Pp. 127-148.

Federal Research Division. 2003. "Terrorists and organized crime groups in the Tri-Border area of South America". Washington: Library of Congress, Central Intelligence Crime and Narcotics Center.

GALLO, Daniel. "Entraron en el país grupos vinculados con Al-Qaeda. Los primeros ingresos se registraron hace siete meses; buscarían reclutar futuros extremistas". La Nación 2005/09/12.

www.lanacion.com.ar/735266

(Consulta: 12-12-2005).

KARMON, Ely. "Hezbollah America Latina: strange group or real threat?" Institute for Counterterrorism. (2006/11/14).

http://www.instituteforcounterterrorism.org/apage/3539.php (Consulta: 22-03-2007).

MONTENEGRO, Silvia y GIMÉNEZ Verónica. La Triple Frontera: globalización y construcción social del espacio. 2006. Miño y Dávila editores. Buenos Aires.

PREVE, E.; SARTHOU, M. “¿Qué esconde el velo de Chuy? El secreto mejor guardado de Chuy”. El Observador 1999/02/07, Montevideo, pp. 3-6.

SANTORO, Daniel, "Las huellas de Bin Laden que la SIDE encontró en la Triple Frontera" EN: Clarín 2001/09/16. Buenos Aires. http://www.clarin.com/diario/2001/09/16/i-00815.htm (Consulta: 17-05-2007).

CUADERNOS JUDAICOS-ISSN: 0718-8749

№26, diciembre 2009 
SCHWARTZ, Alan (editor). 1995. "The struggle is now worldwide", Hizballah and Iranian-Sponsored Terrorism, Anti-Defamation League of B'nai Brith, Special Report, New York, USA.

TORRES SORIANO, Manuel R. "La fascinación por el éxito: el caso de Hezbollah en América Latina". Jihad Monitor Ocasional Paper № 1. 2006. Universidad Pablo de Olavide de Sevilla.

US Department of State. Patterns of Global Terrorism 2002. Washington: National Memorial Institute for the Prevention of Terrorism 2003.

http://www.state.gov/documents/organization/20116.pdf (Consulta: 17-05-2007). 